\title{
The latest update of the advanced crystallography program at NSF's ChemMatCARS
}

\author{
SuYin Grass Wang ${ }^{\mathrm{a}}$, Adam I Stash ${ }^{\mathrm{b}}$ and Yu-Sheng Chen ${ }^{\mathrm{c} *}$ \\ aNSF's ChemMatCARS, The University of Chicago, IL 60439, USA, grass@cars.uchicago.edu \\ bNSF's ChemMatCARS, The University of Chicago, IL 60439, USA, astas@yandex.ru

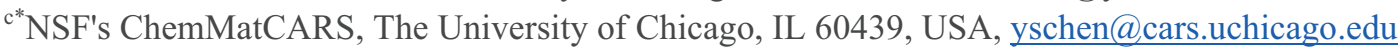

Located at the Advanced Photon Source (APS), NSF's ChemMatCARS addresses the need in the U.S. for facilities and expertise in synchrotron X-ray studies of advanced chemical and materials crystallography, liquid surface/interface scattering, and anomalous small angle x-ray scattering. The NSF's

ChemMatCARS' advanced crystallography program is a dedicated third generation synchrotron X-ray beamline for small molecule. The program includes but not limited to, high-resolution charge density; photo-crystallography; resonant diffraction/Diffraction Anomalous Fine Structure (DAFS) (energy range is from 5 to $70 \mathrm{keV}$ ); structural dynamics; extreme conditions in both pressure and temperature (high P $<10 \mathrm{GPa}$, low T 10K); single micro-crystal diffraction; 3D D-pair distribution function. NSF's ChemMatCARS' advanced crystallography program uses a Huber 3-circle diffractometer equipped with two high dynamic range Pilatus 3X-CdTe-1M and Pilatus $3 \mathrm{X}$ Si $(1 \mathrm{~mm}) 2 \mathrm{M}$ detectors that are optimal for high and low energy single crystal diffraction respectively. This presentation focuses on the experimental setup for 15ID-D hutch and the scientific highlights of the NSF's ChemMatCARS' advanced crystallography program. 